\title{
Antiplatelet therapy following stent deployment
}

\author{
A H Gershlick
}

Stenting might be expected to incur a high complication rate. The stent is a foreign body producing a foreign body reaction (including giant cells) and if made of stainless steel, which carries a negative electrostatic charge, is very thrombogenic. The coronary artery might be expected to be intolerant of such a foreign body especially in the setting of potential reduced flow and following the release of prothrombogenic material (such as is present in atheroma). Initial fears appeared to have been born out when Serruys et al reported a $21 \%$ stent thrombosis rate for the wallstent in $1989^{1}$ and similar results were published by Schatz for the Johnson and Johnson stent. ${ }^{2}$ The obvious answer was anticoagulation, and the more the better. Thus during a second phase of coronary stenting formal anticoagulation was undertaken with warfarin, dextran, dipyridamole, aspirin, and overlapping heparin. ${ }^{3}$ The consequence was little change in thrombosis rate ${ }^{4}$ and an excess of haemorrhagic complications, in particular femoral artery haematoma. This second period of stent anticoagulation lasted from around 1990 to 1994 during which time a number of reports appeared suggesting that stent deployment had been less than optimal ${ }^{56}$ and that the reason for the high thrombosis rate (5-15\%) was the presence of thrombogenic dead spaces between the stent and vessel wall that could be seen on ultrasound examination. ${ }^{7}$ High pressure ballooning post-stent deployment was advocated and intravascular ultrasound encouraged to ensure good stent apposition. Unfortunately at about the same time additional interest in new antiplatelet (rather than antithrombotic) regimens emerged. It has, therefore, been difficult to determine which of the concurrent changes in clinical practice General Hospital, Groby Road, Leicester LE3 9QP, UK

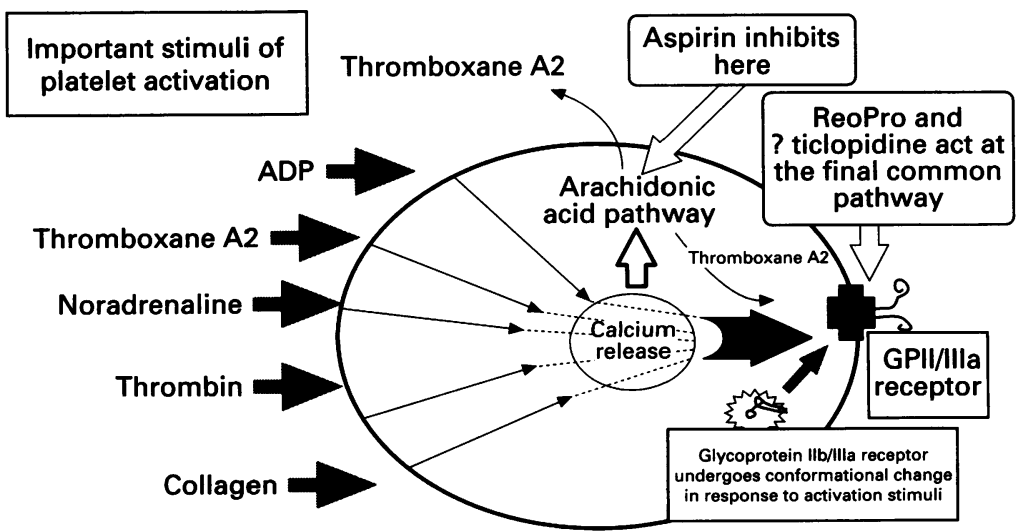

Figure 1 Arachidonic acid pathway. most influenced the reduction in stent thrombosis from $>15 \%$ to about $2 \%$ during this third period of stenting.

The new antiplatelet regimens included ticlopidine. Ticlopidine is the only drug in current clinical practice apart from aspirin that has a true antiplatelet effect. In many ways it has potentially a more potent effect on the platelet than aspirin. Although the exact mechanism for its action is still unclear certain facts are known. First, it needs to be metabolised in the liver to a number of active metabolites for it to have any effect. There are thought to be at least four metabolites that influence platelet function. This has a number of implications. It will not be effective until an adequate plasma concentration of the metabolite(s) has been reached, which may take several days, although this has been disputed and observable antiplatelet effects have been seen within three hours of the first dose. Second, ticlopidine is not a drug that could be effective following local delivery. Because of its site of action it is likely to be more potent than aspirin not least because aspirin and ticlopidine act at different target sites in the platelet.

Aspirin inhibits the platelet through inhibition of the cyclo-oxygenase enzyme, which is an important enzyme in the metabolic pathway that converts arachidonic acid to thromboxane A2. This enzyme catalyses the production of the intermediate pathway products, the endoperoxides. The arachidonic acid pathway normally produces thromboxane $\mathrm{A} 2$, which stimulates other platelets and is a powerful vasoconstrictor. However, the metabolic products of the arachidonic acid pathway, inhibited by aspirin through inhibition of cyclo-oxygenase, are only one way that other platelets are stimulated (fig 1). The final common pathway of platelet stimulation is activation of the glycoprotein IIb/IIIa receptor, a receptor that once stimulated undergoes conformational change to allow it to become available for fibrinogen binding and plateletplatelet aggregation through the fibrinogen bridge (fig 2). While thromboxane A2 does stimulate the glycoprotein IIb/IIIa receptor this is only one of several ways that this receptor can be activated. Other mediators of platelet activation such as thrombin, collagen, noradrenaline, and ADP can activate platelets without activating the arachidonic acid pathway. Through their effect on intraplatelet ionised calcium release, such stimuli cause a direct change in the glycoprotein $\mathrm{IIb} / \mathrm{III}$ receptor conformation and platelet-platelet fibrinogen mediated aggregation. Ticlopidine 


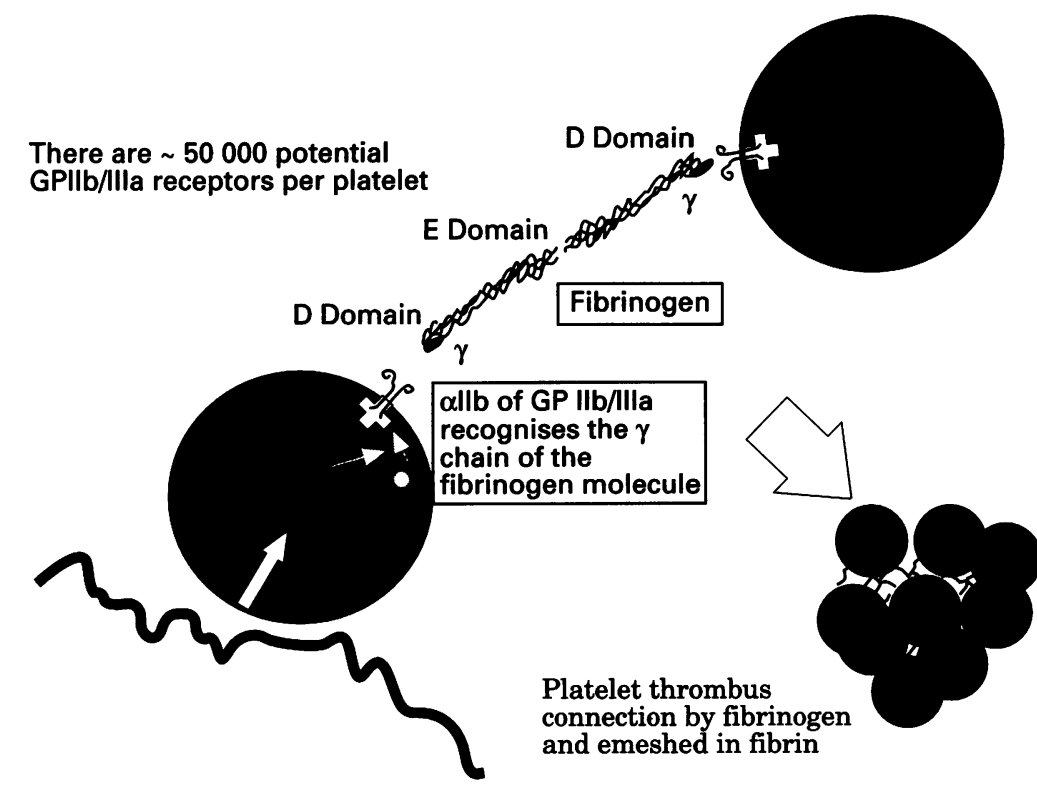

Figure 2 Final common pathway of platelet stimulation.

appears to function by inhibiting the stimulation by ADP of the glycoprotein IIb/IIIa receptor, something aspirin by acting only on the arachidonic acid pathway is unable to do. Inhibition of thromboxane A2 production by aspirin is not synonymous with total inhibition of platelet function.

Thus, in theory at least, ticlopidine could be expected to be a more effective antiplatelet agent than aspirin as it targets the final common pathway. Certainly in trials of transient ischaemic attack, it has been shown to be equally effective to aspirin albeit with a slightly higher and different side effect profile. $^{8}$ Elegant studies by Gawaz and coworkers, using flourescein labelled antibodies against the glycoprotein IIb/IIIa receptor as markers of platelet activation, have clearly shown that platelets are activated following stenting and that the degree of activation is reduced by ticlopidine. ${ }^{9}$

The first question to be asked is whether the combination of ticlopidine and aspirin is better than warfarin for preventing stent thrombosis. A number of published trials clearly demonstrate that the stent thrombosis rate with the antiplatelet combination is about $1-2 \% .{ }^{1011}$ While these studies and others show an overall stent thrombosis rate of about $2 \%$, stenting for bailout incurs a higher risk (5.4\%). In-hospital stay was reduced and side effects from ticlopidine were rare $(1 \cdot 8 \%)$ and reversible after discontinuation of the drug.

Comparison of this data with historical data for patients previously given a warfarin regimen are unhelpful as the indications, techniques, and operator experience have changed. The general consensus is, however, that by focusing on the platelet as an important cause of stent thrombosis there has been a significant impact on outcome. There is a recently published study that directly compared these therapeutic regimens. ${ }^{12}$ The stent thrombosis rate for the warfarin group was $5.4 \%$ and for the antiplatelet agents $0.8 \%$ (rel- ative risk, $0 \cdot 14 ; 95 \%$ confidence interval, $0 \cdot 02-0 \cdot 62$ ). Although it would appear at first glance that this study included all comers, the degree of dissection was not precisely defined between groups so the impact of a pure antiplatelet regimen in truly adverse conditions is still a little unclear. Similar results on the benefit of antiplatelet versus anticoagulant regimens are available from unpublished reports of the FANTASTIC Trial and from a number of other, some small, published trials. ${ }^{13}$ Despite attempts to find a place for warfarin in certain circumstances, such as stenting in the setting of occlusive dissection, oral anticoagulation and the overlapping heparin are no longer used. Some still give subcutaneous heparin with antiplatelet therapy for high risk stenting as described in the earlier French trials.

\section{Aspirin alone}

Is aspirin sufficient as antiplatelet therapy or do you need the combination of aspirin and ticlopidine? There undoubtedly has been a desire to abandon ticlopidine as a treatment after stent implantation. Part of this has been the concern about complications and the initial difficulty in obtaining ticlopidine in the UK. The evidence for giving aspirin alone has been compelling. In Colombo's study with antiplatelet treatment and high pressure intravascular ultrasound, aspirin alone appeared almost as good as the combination of aspirin and ticlopidine with stent thrombosis rates of $1.4 \%$ and $0.8 \%$, respectively, although the difference may have failed to achieve significance because of sample size. In a randomised trial by the same group ${ }^{14} 226$ patients received either aspirin alone $(n=103)$ or a combination of short term aspirin and ticlopidine $(\mathrm{n}=$ 123). At one month the stent thrombosis rate was $2.9 \%$ in the aspirin group and $0.8 \%$ in the combination group, again a difference that was not significant. The authors acknowledge however that the combination of a low incidence of the end point and small numbers randomised may again have been responsible for the difference failing to achieve significance. Other randomised studies have also tended to have small numbers.

Larger trials are being done; thus we await the full publication of the MUST, STARS, and MUSIC studies. There has been preliminary presentation of results from these studies and the MUST data confirms the low incidence of stent thrombosis with antiplatelet (aspirin and ticlopidine) therapy without the need to resort to intravascular ultrasound (IVUS) guidance but with high pressure ballooning.

STARS is a completed study using the Johnson \& Johnson (Palmaz-Shatz) stent in which patients were randomised to aspirin, aspirin and warfarin, or aspirin and ticlopidine. Post-stent high pressure was used and IVUS use was optional. There was no pretreatment with ticlopidine. The trial group consisted of 55 sites in the US who have randomised 1650 patients to one of the three groups $(553,544$, and 550 patients, respec- 
tively). The primary end point was clinical subacute closure within 30 days (hierarchical composite of death, emergency bypass surgery, $Q$ wave myocardial infarction, and subacute closure with repeat revascularisation within 30 days). Early data indicates that the post-stent mean deployment pressure was $16.8 \mathrm{~atm}$ and two thirds of patients received single stents (the mean lesion length being $10.5,9.9$, and $10.3 \mathrm{~mm}$ in the three groups, respectively). The as yet unpublished data indicates that the end point was reached in $2.4 \%$ of the aspirin and warfarin group, but in only $0.6 \%$ of the aspirin plus ticlopidine group. However this point was reached in $3.6 \%$ of the aspirin alone group $(P=0.001)$. Stent thrombosis occurred in $0.6 \%$ of the aspirin and ticlopidine group but in $2.9 \%$ of the aspirin alone group, and $2.4 \%$ of the aspirin and warfarin group $(P=0.001)$.

Early unpublished reports from the MUSIC Trial (IVUS guided, aspirin only) suggests that stent thrombosis was also low and about the same as the ticlopidine plus aspirin group from the STARS trial $(\sim 2 \%)$. This, together with the STARS data, could suggest that if one wants to use aspirin then IVUS guided stent deployment may be necessary, whereas the addition of ticlopidine allows a low thrombosis rate with high pressure ballooning but without the need for IVUS directed stenting. Vessel size may be a critical issue and it is to be hoped that once these trials are published in full a better idea of who can receive aspirin and high pressure ballooning without need for IVUS (perhaps those with vessels over $3.2 \mathrm{~mm}$ diameter) will become evident.

\section{Conclusions}

Aspirin and ticlopidine act at different parts of the various intraplatelet activation pathways. It has been recognised for some time that aspirin is limited in its effect as an antiplatelet agent, particularly in circumstances likely to be present when stents thrombose, such as generated thrombin and collagen from disrupted atheroma. As it became clear that platelets were central to stent thrombosis and antiplatelet therapy has been shown to be the mainstay of therapy, the limitations of aspirin have been forgotten and aspirin alone has been advocated. Once tested in a trial large enough to demonstrate a difference, such as STARS, the extra benefit of ticlopidine becomes apparent. The fact that such a difference could not be shown with smaller trials says something about the biological importance of the difference, particularly as this needs to be set against the extra complications associated with ticlopidine $(0.4 \%$ transient neutropenia in STARS). Stent thrombosis is such a potential disaster ( $50 \%$ myocardial infarction rate) however that the use aspirin and ticlopidine in combination should be recommended. Better antiplatelet agents than ticlopidine such as clopidogrel have been developed. Future trials comparing clopidogrel and aspirin with ticlopidine and aspirin would be worth considering. Aspirin alone may be acceptable in patients in whom the stent deployment is IVUS directed or in those with large vessels or in whom low risk de novo stenting has been undertaken with heparin coated stents. ${ }^{15}$

Whether all the studies purporting to include patients at high risk of stent thrombosis truly have included such high risk patients, and whether other groups such as those with occlusive dissection in small $(<2.8 \mathrm{~mm})$ vessels will truly be protected by an oral antiplatelet combination of aspirin and ticlopidine is yet to be determined. Certainly such patients had a excess risk of thrombosis in the French registry data. A drug eluting truly antithrombotic stent is likely to be developed for these patients. Such agents will need to act, like ticlopidine, at the final glycoprotein IIb/IIIa receptor site.

1 Serruys PW, Strauss BH, Beatt KJ, et al. Angiographic follow up after stent placement of a self expanding coronary artery stent. $N$ Engl f Med 1991;324:13-17.

2 Schatz RA, Baim DS, Leon M, et al. Clinical experience with the Palmaz-Schatz coronary stent. Initial results of a multicentre study. Circulation 1991;83:148-61.

3 Brack MJ, Hubner PJ, Gershlick AH. Anticoagulation after Brack MJ, Hubner PJ, Gershlick AH. Antico
stent insertion. Br Heart $₹$ 1994;72:294-6.

4 Nath FC, Muller DWM, Ellis SG, et al. Thrombosis of a flexible coil coil stent: frequency, predictors, and clinical flexible coil coil stent: frequency, predictors,
outcome. ₹ Am Coll Cardiol 1993;21:622-7.

5 Serruys PW, Di Mario C. Who was thrombogenic: the stent or the doctor? Circulation 1995;91:1891-3.

6 Mudra H, Blasini R, Regar E, et al. Intravascular ultrasound assessment of the balloon-expandable PalmazSchatz stent. Coronary Artery Dis 1993;4:2026-34.

7 Nakamura S, Colombo A, Gaglione A, et al. Intracoronary ultrasound observations during stent implantation. Circulation 1994;89:2026-34.

8 Haynes RB, Sandler RS, Larson EB, et al. A critical appraisal of ticlopidine, a new antiplatelet agent. Arch Intern Med 1992;152:1376-80.

9 Neuman FJ, Gawaz M, Ott I, et al. Prospective evaluation of haemostatic predictors of sub-acute stent thrombosis after coronary Palmaz-Schatz stenting. $\mathfrak{F} \mathrm{Am}$ Coll Cardiol 1996;27:15-21.

10 Colombo A, Hall P, Nakamura S, et al. Intracoronary stenting without anticoagulation accomplished with intravascuiar

11 Lablanch J-M, McFadden EP, Bonnet J-L, et al. Combined antiplatelet therapy with ticlopidine and aspirin. Eur antiplatelet therapy with
Heart $₹ 1996 ; 17: 1373-80$.

12 Schomig A, Neuman FJ, Kastrati A, et al. A randomised comparison of antiplatelet therapy after the placement of coronary stents. NEngl f Med 1996;334:1084-9.

13 Saito S, Hosokawa Fg, Kim K, et al. Primary stent implantation with out coumadin in acute myocardial infarction. $f$ Am Coll Cardiol 1996;28:74-81.

14 Hall P, Nakamura S, Maiello L, et al. A randomised comparison of combined ticlopidine and aspirin therapy versus aspirin alone after successful intravascular ultrasoundguided stent implantation. Circulation 1996;93:215-22.

15 Serruys PW, Emanuelsson $\mathrm{H}$, van der Giessen W, et al Heparin-coated Palmaz-Schatz stents in human coronary arteries. Early outcome of the Benestent II pilot study. Circulation 1996;93:412-22. 